\title{
Tail states in superconductors with weak magnetic impurities
}

\author{
I. Vekhter ${ }^{\mathrm{a}, 1}$, A. V. Shytov ${ }^{\mathrm{b}}$, I. A. Gruzberg ${ }^{\mathrm{c}}, \mathrm{A}$. V. Balatsky ${ }^{\mathrm{a}}$ \\ ${ }^{a}$ Theoretical Division, MS B262, Los Alamos National Laboratory, Los Alamos, NM 87545 \\ ${ }^{\mathrm{b}}$ Kavli Institute for Theoretical Physics, University of California, Santa Barbara, CA 93106 and \\ L. D. Landau Institute for Theoretical Physics, 2 Kosygin St., Moscow, Russia 117334 \\ ${ }^{\mathrm{c}}$ Department of Physics, Massachusetts Institute of Technology, Cambridge, MA 02139
}

\begin{abstract}
We analyse the behavior of the density of states in a singlet $s$-wave superconductor with weak magnetic impurities in the clean limit by using the method of optimal fluctuation. We show that the density of states varies as $\ln N(E) \propto$ $-\left|E-\Delta_{0}\right|^{(7-d) / 4}$ near the mean field gap edge $\Delta_{0}$ in a $d$-dimensional superconductor. The optimal fluctuation in $d>1$ is strongly anisotropic. We compare the density of states with that obtained in other recent approaches.
\end{abstract}

Studies of spectral properties of disordered superconductors remain an active area of research as they help advance our understanding of the competition between disorder and interactions. Recent years witnessed a renewed interest in the behavior of the density of states (DOS) in singlet $s$-wave superconductors with magnetic impurities. Such impurities are pairbreaking, and are characterized, in the weak scattering limit, by the spin flip scattering time, $\tau_{s}$. In the self-consistent Born approximation (SCB) [1], the dimensionless parameter controlling the suppression of the single particle spectral gap, $\Delta_{0}$, is $\Delta \tau_{s}$, where $\Delta$ is the amplitude of the superconducting order parameter. In this paper we consider the clean limit, $\Delta \tau_{s} \gg 1$, where the SCB approach yields a finite spectral gap, $\Delta_{0} \approx \Delta$, with the $\operatorname{DOS} N(E)=0$ at energies $E<\Delta_{0}$.

It was argued in Ref.[2] that rare regions where local impurity concentration is high enough to locally destroy superconductivity lead to a finite density of states at the Fermi level. The argument was similar to the method of optimal fluctuation (OF), well known from studies of doped semiconductors [3]: in averaging over all the realizations of the impurity distribution, the probability of finding the realization which locally destroys the gap determines the residual DOS. Later, Lamacraft and Simons [4] considered in detail the energy dependence of the DOS below the mean field gap

\footnotetext{
1 Corresponding author. E-mail: vekhter@lanl.gov
}

in a dirty superconductor, where the scattering rate due to potential scattering greatly exceeds the spin-flip pairbreaking scattering rate, $1 / \tau_{s}$.

Very recently we analysed the subgap DOS in a clean $s$-wave superconductor, where the spin-flip scattering is dominant [5]; we argued that at least in some cases this limit is relevant experimentally. Here we briefly review the results of Ref.[5] and then present a more detailed comparison of the DOS obtained in Refs. [2,4,5].

We consider a mean field hamiltonian

$\widehat{H}=\widehat{\xi} \tau_{3}+\Delta(\mathbf{r}) \tau_{1} \sigma_{2}+\widehat{U}$,

where $\widehat{\xi}=-\nabla^{2} /(2 m)-\mu, \mu$ is the chemical potential, $\tau_{i}$ and $\sigma_{i}$ are the Pauli matrices in the particle-hole and the spin space respectively. The potential due to magnetic impurities $\widehat{U}=\mathbf{U}(\mathbf{r}) \cdot \mathbf{s}$, where $\mathbf{s}$ is the electron spin operator, $\mathbf{U}(\mathbf{r})=\sum_{i} J \mathbf{S}_{i} \delta\left(\mathbf{r}-\mathbf{r}_{i}\right), J$ is the exchange constant, and $\mathbf{S}_{i}$ is the impurity spin at a site $i$.

For an energy, $E<\Delta_{0}$, OF is the most probable configuration of impurities that creates a state at $E$, and therefore contributes the most to the DOS [3]. OF provides nonperturbative corrections to the DOS determined in the framework of SCB. In essentially all the energy range below the gap the size of the OF is significantly greater than the distance between impurities, so that the exact impurity potential can be replaced by a smooth function, and its probability density is well approximated by a Gaussian [3] with a width $U_{0}^{2}=$ 
$n_{i m p} J^{2} S(S+1) / 3$, so that $\tau_{s}^{-1}=2 \pi N_{0} U_{0}^{2}$, where $n_{i m p}$ is the impurity concentration, and $N_{0}$ is the normal state DOS. The DOS is then given by $\ln \left[N(E) / N_{0}\right] \approx$ $-\mathrm{S}\left[\mathbf{U}_{o p t}\right]$, where $\mathrm{S}\left[\mathbf{U}_{o p t}\right]$ is obtained by minimizing

$\mathrm{S}[\mathbf{U}]=\frac{1}{2 U_{0}^{2}} \int d^{d} \mathbf{r} \mathbf{U}^{2}(\mathbf{r})+\lambda(\mathrm{E}[\mathbf{U}]-E)$

with respect to both the potential $\mathbf{U}$ and the Lagrange multiplier $\lambda$. We assume OF to be ferromagnetic [5], so that $\mathrm{E}[\mathbf{U}]=\left\langle\Psi\left|\widehat{\xi} \tau_{3}+\Delta_{0} \tau_{1}+U\right| \Psi\right\rangle$ where $\Psi$ is the normalized spinor wave function of the particle in the $\mathrm{OF}$, and $U(x)=-\lambda U_{0}^{2}\left(\Psi^{\star}(x) \Psi(x)\right)$ is now scalar.

In $d=1$ we find $\left(\epsilon=|E| / \Delta_{0}\right)$

$$
\begin{aligned}
& \frac{U(x)}{2 \Delta_{0}}=-\frac{1-\epsilon^{2}}{\epsilon+\cosh \left(2 x \sqrt{1-\epsilon^{2}} / \xi_{0}\right)}, \\
& \mathrm{S}_{0} \equiv \mathrm{S}\left[U_{\text {opt }}\right]=8 \pi\left(\Delta_{0} \tau_{s}\right)\left[\sqrt{1-\epsilon^{2}}-\epsilon \arccos \epsilon\right],
\end{aligned}
$$

where $\xi_{0}=v_{F} / \Delta_{0}$ is the coherence length and $v_{F}$ is the Fermi velocity. The size of the OF, $L_{x} \simeq \xi_{0} / \sqrt{1-\epsilon^{2}} \geq$ $\xi_{0}$, and the depth of the potential $|U| \leq 2 \Delta_{0}$. In particular, near the gap edge, $1-\epsilon \ll 1$, when $|U| \ll \Delta_{0}$ and $L_{x} \gg \xi_{0}$, we find $\mathrm{S}_{0} \simeq(8 \pi / 3)\left(\Delta_{0} \tau_{s}\right)\left(1-\epsilon^{2}\right)^{3 / 2}$, which allows for a simple interpretation. In an OF of depth $U$ and size $L_{x}$ the energy of the bound state is $E \simeq$ $U+\Delta_{0}+v_{F}^{2} / L_{x}^{2} \Delta_{0}$, subject to optimization of Eq.(2). This means $\left|E-\Delta_{0}\right| \sim v_{F}^{2} / L_{x}^{2} \Delta_{0} \sim|U|$, from which an estimate for $\mathrm{S}_{0} \simeq L_{x} U^{2} / U_{0}^{2} \sim\left(\Delta_{0} \tau_{s}\right)\left(1-\epsilon^{2}\right)^{3 / 2}$ follows immediately.

In $d>1$ and for $1-\epsilon \ll 1$ we must compare the action for the isotropic OF with $L \sim \xi_{0} / \sqrt{1-\epsilon^{2}}$ (when kinetic energy is $v_{F} / L$ ), and for an anisotropic OF with $L_{x} \sim L$, and transverse size $L_{t} \sim\left(L_{x} / k_{F}\right)^{1 / 2}$ (corresponding to a wave function $\Psi(\mathbf{r})=\exp \left(i k_{F} x\right) \Phi(x, \mathbf{y})$, where $\Phi$ is a slowly varying function, so that $\left.1 /\left(m L_{t}^{2}\right) \sim v_{F} / L_{x}\right)$. The anisotropic OF is favored by a factor $\left(E_{F} / \Delta_{0}\right)^{(d-1) / 2}(1-\epsilon)^{-(d-1) / 4}$ and gives [5]

$\mathrm{S}_{0} \simeq L_{x} L_{t}^{d-1} \frac{U^{2}}{U_{0}^{2}} \simeq\left(\Delta_{0} \tau_{s}\right)\left(\frac{E_{F}}{\Delta_{0}}\right)^{\frac{d-1}{2}}(1-\epsilon)^{\frac{7-d}{4}}$.

We now compare this result with that of Ref.[4] for the diffusive propagation of the states within OF. It is clear that even in the clean case considered here, with dilute magnetic impurities, a transition to the diffusive regime occurs when the size of OF $L \geq v_{F} \tau_{s}$, or $1-\epsilon \leq\left(\Delta_{0} \tau_{s}\right)^{-2}$. In our notations the result of Ref.[4] for $\Delta_{0} \tau_{s} \gg 1$ reads $\mathrm{S}_{D}=$ $\left(\Delta_{0} \tau_{s}\right)^{5 / 3}\left(E_{F} / \Delta_{0}\right)^{d-1}(1-\epsilon)^{(6-d) / 4}$. Consequently, at the crossover point the action from Eq. (5) is smaller, $\mathrm{S}_{D} / S_{0} \simeq\left(E_{F} / \Delta_{0}\right)^{(d-1) / 2}\left(\Delta_{0} \tau_{s}\right)^{7 / 6} \gg 1$, and the OF found here corresponds to a greater DOS. As the size of the OF increases even further, the anisotropic fluctuation becomes insupportable due to diffusive motion. Nonetheless, even for an isotropic fluctuation, we find $\mathrm{S}_{D} / S_{\text {iso }} \simeq\left(\Delta_{0} \tau_{s}\right)^{2 / 3}(1-\epsilon)^{(d-2) / 4}$, which, at the crossover, gives $\left(\Delta_{0} \tau_{s}\right)^{(10-3 d) / 6} \gg 1$ for $d \leq 3$. Consequently, the OF discussed here still yields a higher DOS than that for purely diffusive motion. Therefore we expect that the structure of the OF near the crossover between the ballistic and diffusive regimes still resembles closely that given by us above, and that our results remain at least qualitatively valid there.

Balatsky and Trugman [2] considered only the DOS at $E=0$, where they needed a large volume fluctuation, $V \geq \xi^{d}$, which is less probable and yields lower DOS than that of Eq. (5). However, in the same spirit we should investigate whether local suppression of the gap from $\Delta_{0}$ to $E$ due to a large number of impurities with uncorrelated spins (as opposed to a ferromagnetic OF above) is advantageous. For $1-\epsilon \ll 1$ the local pairbreaking rate, $\gamma$, needed to reduce the gap to $E$ is $\gamma \tau_{s} \approx 1+(1-\epsilon)\left(\Delta_{0} \tau_{s}\right)^{2 / 3}$, and the volume of the region has to be at least equal to that of the anisotropic OF to avoid high kinetic energy cost (this is an underestimate since it ignores proximity coupling to bulk). In that case we obtain the optimal action $\mathrm{S}_{B T} / \mathrm{S}_{0} \approx$ $\left(\Delta_{0} \tau_{s}\right)^{1 / 3}\left(E_{F} / \Delta_{0}\right) \bar{c}$, where $\bar{c}=n_{i m p} \lambda_{F}^{d}$ is the atomic concentration of impurity atoms. As a result, for realistic values of $\bar{c}$ and clean samples $\mathrm{S}_{B T} \gg \mathrm{S}_{0}$, and the DOS given by the action in Eq.(5) is higher.

We conclude that the action obtained in our approach gives the most optimal fluctuation and the highest DOS compared to other analyses.

Acknowledgements This research was supported by the DOE under contract W-7405-ENG-36, by the NSF Grant PHY-94-07194, and by Pappalardo Fellowship. We are grateful to the ITP Santa Barbara and Aspen Center for Physics for hospitality and support.

\section{References}

[1] A. A. Abrikosov and L. P. Gor'kov, Sov. Phys. JETP 12 (1961) 1243.

[2] A. V. Balatsky and S. A. Trugman, Phys. Rev. Lett. 79 (1997) 3767.

[3] For a review, see I. M. Lifshits, S. A. Gredeskul, and L. A. Pastur, Introduction to the theory of disordered systems, (John Wiley \& Sons, New York, 1988).

[4] A. Lamacraft and B. D. Simons, Phys. Rev. Lett. 85 (2000) 4783; Phys. Rev. B 64 (2001) 014514.

[5] A. V. Shytov_ I Vekhter, I A. Gruzberg, and A. V. Balatsky, cond-mat/0206183. 